\title{
Improvement of the Lewis-Abegg-Octet Rule Using an "Even-Odd" Rule in Chemical Structural Formulas: Application to Hypo and Hyper-Valences of Stable Uncharged Gaseous Single-Bonded Molecules with Main Group Elements
}

\author{
Geoffroy Auvert \\ CEA-Leti 17 Avenue des martyrs F38000, Grenoble, France \\ Email: Geoffroy.auvert@grenoble-inp.org
}

Received 12 February 2014; revised 10 March 2014; accepted 17 March 2014

Copyright (C) 2014 by author and Scientific Research Publishing Inc.

This work is licensed under the Creative Commons Attribution International License (CC BY).

http://creativecommons.org/licenses/by/4.0/

(c) (i) Open Access

\section{Abstract}

As Lewis proposed his octet rule, itself inspired by Abegg's rule, that a molecule is stable when all its composing atoms have eight electrons in their valence shell, it perfectly applied to the vast majority of known stable molecules. Only a few stable molecules were known that didn't fall under this rule, such as $\mathrm{PCl}_{5}$ and $\mathrm{SF}_{6}$, and Lewis chose to leave them aside at the time of his research. With further advances in chemistry, more exceptions to this rule of eight have been found, usually with the central atom of the structure having more or less than eight electrons in its valence shell. Theories have been developed in order to modify the octet rule to suit these molecules, defining these as hyper- or hypo-valent molecules and using other configurations for the electrons. The present paper aims to propose a representation rule for gaseous single-bonded molecules that makes it possible to reconcile both; molecules following the octet theory and those which do not. In this representation rule, each element of the molecule is subscripted with two numbers that follow a set of simple criteria. The first represents the number of valence electrons of the element; while the second is calculated by adding the first number to the number of the element's covalent bonds within the molecule. The latter is equal to eight for organic molecules following the octet rule. Molecules being exceptions to the octet rule are now encompassed by this new even-odd rule: they have a valid chemical structural formula in which the second number is even but not always equal to eight. Both rules-octet and even-odd-are discussed and compared, using several wellknown gaseous molecules having one or several single-bonded elements. A future paper will discuss the application of the even-odd rule to charged molecules. 


\section{Keywords}

\section{Octet Rule, Hypervalence, Hypovalence, Even-Odd, Rule, Electron-Pair, Single Bond, Molecule, Chemistry}

\section{Introduction}

A chemical structural formula is a 2D representation of a molecule with elements symbolized by letters and bonds between two neighbor atoms by a line. This representation is necessary to describe a more precise view of the molecular structure and geometrically discriminate between various isomers having the same chemical formula. William Higgins [1] and J. Crow Brown [2] were the pioneers of this representation. Between 1900 and 1930, with the development of the periodic table [3] and the discovery of the electron, Abegg [4], Lewis [5] and Langmuir [6] have proposed modern bases for structural formulas. Abegg suggested that the valence is frequently eight for an element connected to other elements. Lewis proposed that the bond between elements uses multiple electron pairs. To represent these pairs, Lewis imagined a dot diagram where shared electrons are clearly identified. He proposed furthermore that the octet in Abegg's rule corresponds to saturation in the outer shell of the element. Langmuir in turn described a chemical bond as a connection of an atom only with their nearest neighboring atoms and named "covalent bond" the electron-pair described by Lewis. Since then, the Abegg-Lewis-octet rule is largely used to represent structural formulas of molecules.

Nevertheless, even Lewis noticed that molecules incompatible with the octet rule do exist, although he decided at the time that they were of no great importance. More exceptions have been found since then and theory was required to explain their existence. Models to predict molecular geometry of these so called hypo- or hypervalent molecules are still being investigated. R. J. Gillespie [7] [8] has for instance proposed the idea of Valence Shell Electron Pair Repulsion, in which local charges appear in specific cases; unfortunately without expressing a formal rule. Moreover, no proposition has yet been made to link hypo-valent molecules with the Lewis-Abegg-octet rule.

The present paper aims to propose a more general rule to draw structural formulas that will be named the even-odd rule. It will be shown that the proposed rule is applicable to molecules that follow the octet rule as well as hypo- and hyper-valent molecules. The application of the proposed even-odd rule is applied to several wellknown gaseous uncharged molecules with atoms from the main group in the periodic table.

Note that all molecules are written like $\mathrm{H} 2 \mathrm{O}$ instead of $\mathrm{H}_{2} \mathrm{O}$ for a better computer compatibility.

\section{Even-Odd Rule}

The even-odd rule, proposed for chemical structural formula of gaseous uncharged single-bonded molecules is as follow:

- One element of the molecule is connected only to each of its first neighbor by only one covalent bond represented by a single line.

- An atom described by the letters of its element, is associated with two numbers.

- The left side number is the number of electrons in the valence shell of the element as it is written for the main group in the periodic table. it can range from one for elements like sodium (Na) up to eight for a noble gas like Argon (Ar)

- The right side number, always an even number, is the addition of the left side number with the amount of single bonds around the element.

- The number of single bonds around the element is even for an element having an even amount of valence electrons and odd in the other case.

- The smallest number of single bonds is one for an odd element (left side number) and two for an even one.

- The right side number can go up to twice the left side number corresponding to the maximum of covalent bonds for this element.

- When the right side number is equal to eight, the element follows the Lewis-octet rule.

- When each element of a molecule has this Lewis-octet property, the molecule is in agreement with the Lewis-octet rule. 
The group of molecules following this even-odd rule and having only covalent bonds i.e. with electron pairs, may be named as Electron-Pair (EP) molecules. Also, from this proposed rule, it seems possible that all the Lewis-octet gaseous molecules only having right-side numbers equal to 8, are included in this group of EP molecules.

\section{Application to Uncharged Single-Bonded Gaseous Molecules}

In the following, the validity of the even-odd rule is tested using well-known gaseous molecules. As hundreds of thousands of them exist, the test is, in this paper, limited to gaseous uncharged single-bounded molecules of the main group of elements of the periodic table. In Table 1 and Table 2 are shown several chemical structural formulas for uncharged molecules. Table 1 shows several single-bonded gaseous molecules following the Lewis-octet rule and Table 2 lists gaseous molecules following the even-odd rule but not the Lewis-octet rule.

In Table 1, the first structural formula is for the di-hydrogen gaseous molecule. The left side number is one corresponding to the number of electrons of this element. The right side number corresponds to the addition of one valence electron and one covalent bond allowing the di-hydrogen molecule to be stable in the gas phase. This follows the Lewis rule for hydrogen.

The second structural formula in Table 1, is for the di-chlorine gaseous molecule. The left side number is 7 as in the periodic table. According to the even-odd rule this element may have a number of covalent bonds being 1 , 3,5 or 7. As there is only one covalent bond, the left side number is equal to eight corresponding to the di-chlorine gaseous molecule, which is also in agreement with the Lewis octet rule. The other molecules in Table 1 are using the same procedure to write each corresponding structural formula following the Lewis-octet rule. For example, piperazine molecule C4H10N2 has Carbon and Nitrogen elements having a right side number of eight.

With a global view of Table 1, no element from column 2 and 3 of the periodic table are present. Also, no element having more than 4 single-bonds are in this list and most molecules are mainly organic compounds.

As these molecules are with covalent bonds, they can be classified as EP molecules.

In Table 2, structural formulas are drawn using the same procedure including inorganic molecules and elements from column 2 and 3 of the periodic table. The first molecule is Lithium gas. The formula looks the same as the di-hydrogen molecule of Table 1 . As for hydrogen, the left side number is one, in agreement of the classification table, but a difference appears in the right side number. As only one covalent bond is possible, the right side number is only two. It should have been 8 to be in agreement with Lewis-octet rule. This Li2 gaseous molecule commonly classified as a hypovalent molecule is only following the even-odd rule.

Other molecules in Table 2 are drawn using the same procedure to write each corresponding structural formula. The structure always has at least one right side number not equal to eight. When the right side number is below 8 , the molecule is a hypovalent molecule and above eight, they are hypervalent molecules. These molecules are only following the even-odd rule and they can be considered as EP molecules due to their covalent bonding property.

It may be interesting to observe the last two molecules in Table 2. Their right side number is far above the octet number and does not follow the octet rule. So they are currently classified as hypervalent molecules but they easily fall under the even-odd rule proposed in this paper.

\section{Discussion}

Globally in Table 1, all molecules have a right side number equal to eight. They can be classified in a group named octet molecules. In the second, about $50 \%$ are hypovalent molecule and $50 \%$ hypervalent. They are in agreement only with the even-odd rule. They can be classified in a group named non-octet molecules.

From this observation, the even-odd rule includes in the same field of EP molecules: octet molecules, and non-octet molecules.

\section{Conclusions}

For single-bonded uncharged molecules studied here, the octet rule is too restrictive and all of them are in agreement with the even-odd rule proposed in this paper. The classification of other well-known molecules using the even-odd rule is showing that this rule is more practical and extendable to a larger number of existing 
Table 1. Compatibility of even-odd rule with Lewis-octet rule. Names, formulas and structural formulas of uncharged single-bonded molecules are classified by the atoms number. All molecules, stable in the gas phase, are composed of atoms from the main group of elements and are interconnected to each of their first neighbor by only one covalent bond. All left side numbers come from Mendeleev periodic table and all right side numbers are equal to eight except for hydrogen with a left-side number of 2 according to Lewis-octet rule.

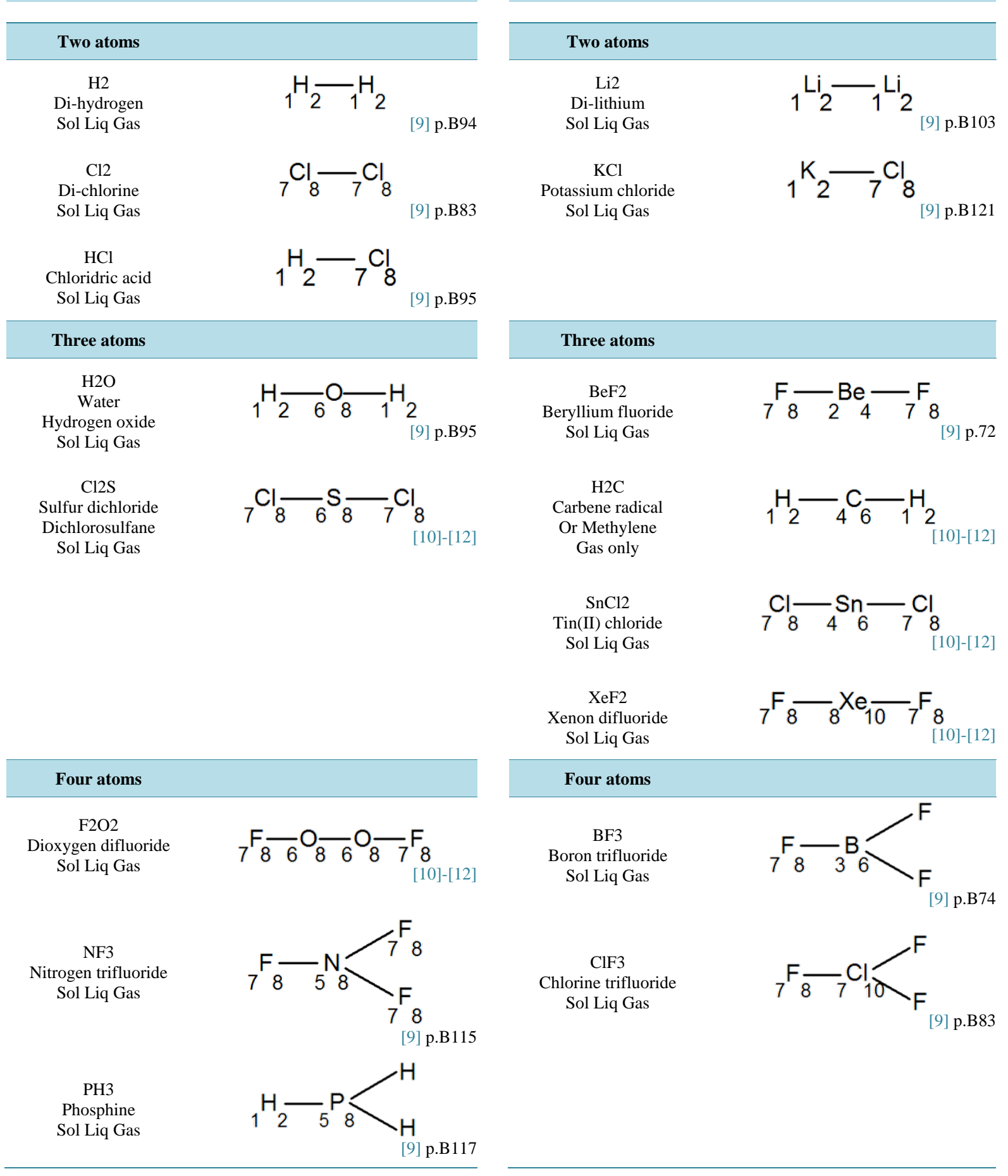

Table 2. Molecules following only the even-odd rule. Names, formulas and structural formulas of uncharged single-bonded molecules are classified by the atoms number. All molecules are stable in the gas phase and all atoms belong to the main group of elements and are interconnected to each of their first neighbor by only one covalent bond. All left side numbers originate from Mendeleev periodic table and are from 1 for Lithium (Li) to 8 for Xenon (Xe) and right side numbers from 2 to 14, are calculated using the even-odd rule defined above. 


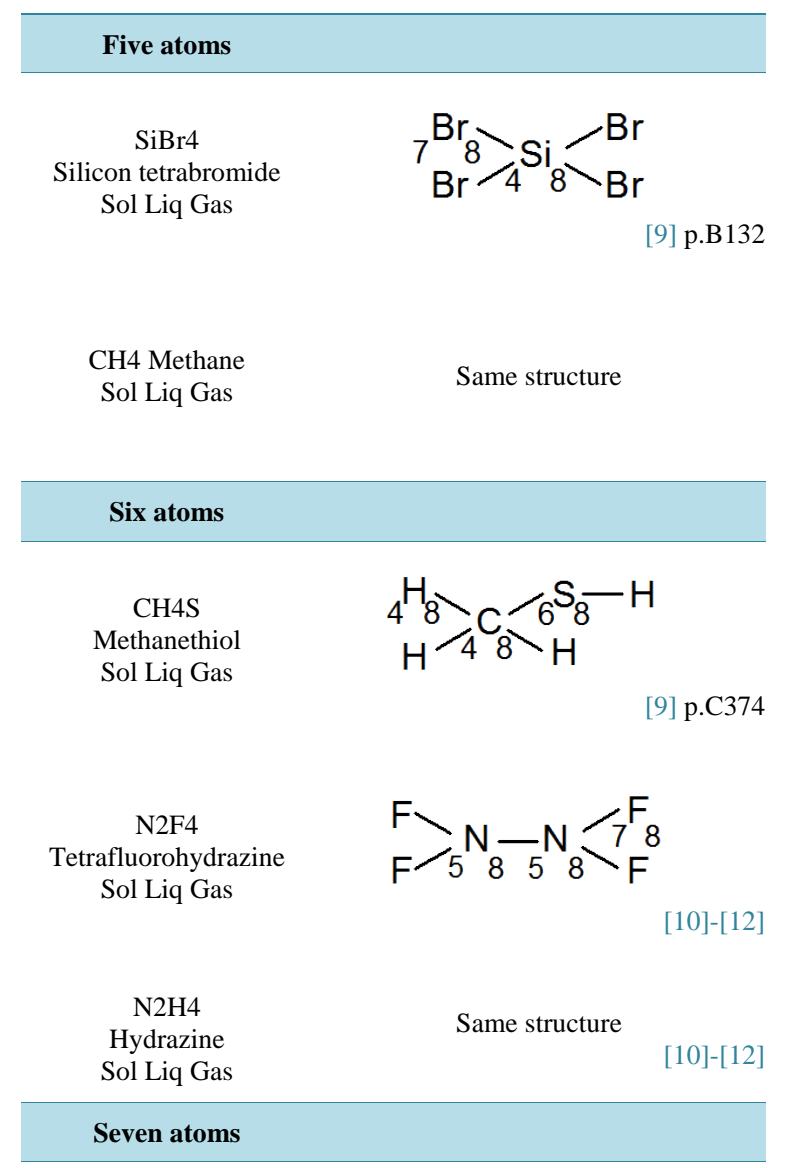

$\mathrm{C} 2 \mathrm{H} 4 \mathrm{O}$
$\mathrm{H} 4 \mathrm{C} 2 \mathrm{O}$
Ethylene oxide

Sol Liq Gas

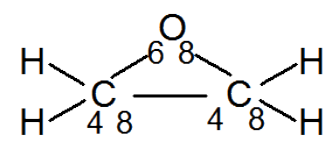

[9] p.C287, [13]

CH4O2

Methanediol

Sol Liq Gas

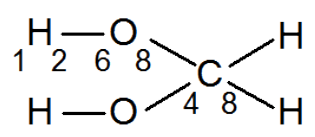

[9] p.C339

CH3NH2

Methylamine

Sol Liq Gas

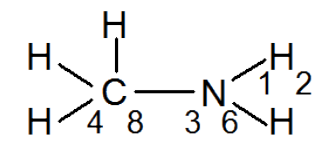

[9] p.C365

Eight atoms

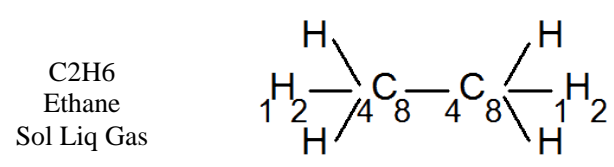

[9] p.C284

\section{Five atoms}

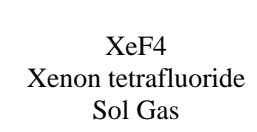

${ }_{F} \mathrm{~F}_{8} \succ_{8} \mathrm{Xe}_{12} \underset{12}{>}{ }_{7} \mathrm{~F}_{8}$

[10]-[12]

SF4

Sulfur tetrafluoride

Sol Liq Gas

${ }_{F} \mathrm{~F}_{8}>{ }_{6} \mathrm{~S}_{10}>{ }_{7} \mathrm{~F}_{8}$

[9] p.B143

\section{Six atoms}

\section{B2F4 \\ Diboron tetrafluoride \\ Sol Liq Gas}

$\mathrm{F}>3 \mathrm{~B}_{6}-\mathrm{B}_{6}>\mathrm{F}$

Sol Liq Gas

PF5

Phosphorus pentafluoride

Sol Liq Gas

[9] p.B117

$$
{ }_{\mathrm{F} /{ }_{5} \mathrm{I}_{\mathrm{F}}}^{\mathrm{F}}-\mathrm{F} 8
$$

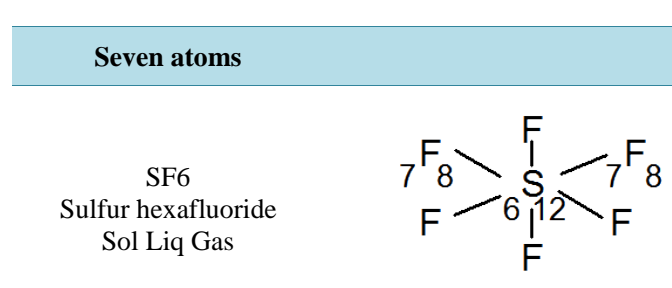

[9] p.B143

XeF6

Xenon hexafluoride

Sol Liq Gas

[12]

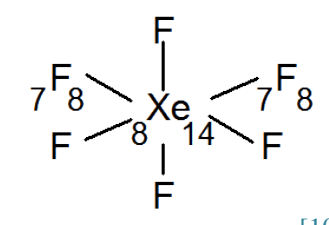

[10]-[12]

BH3O3
Boric acid

[9] p.B74

[9] p.B117

(13


More than eight

C3H6O2

Dioxolane

Sol Liq Gas

[9] p.C271

C4H10N2

Piperazine

Sol Liq Gas

[9] p.C434<smiles>CN1CCN(C)CC1</smiles>

C6H12

Cyclohexane

Sol Liq Gas

[12]

[9] p.C254<smiles>C1CCCCC1</smiles>

C3H2ClF5O

Isoflurane

Sol Liq Gas

[10]-[12]

C2H8N2

1,1-Dimethylhydrazine Sol Liq Gas

C2H8N2

1,2-Dimethylhydrazine Sol Liq Gas

[9] p.C337
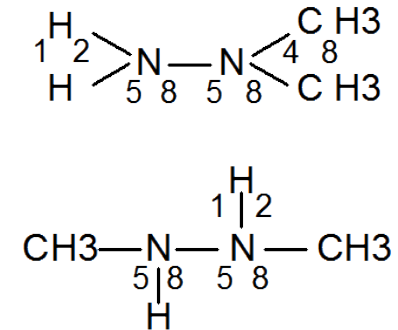

$\mathrm{H}_{4} \mathrm{C}_{8}-{ }_{4} \mathrm{C}_{8} \mathrm{H} 2$

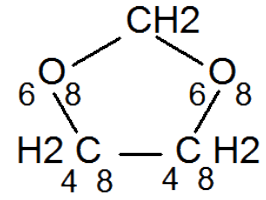

S2F10

Disulfur decafluoride Sol Liq Gas
[9] p.B143

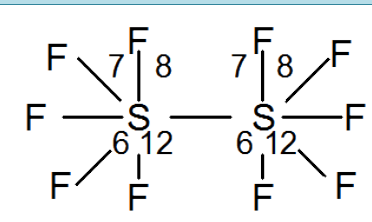

More than eight

molecules like hypo and hypervalent molecules than those following only the octet rule.

These neutral molecules form a group of electron-pair molecules including octet molecules and non-octet molecules as hypo and hypervalent molecules. The next step will be a comparison of the Lewis-octet rule with the even-odd rule for charged electron-pair molecules.

In this paper, gaseous molecules are uncharged and single-bonded and it will be interesting to analyze with the even-odd rule, the structural formula of molecules having multiple-bonds in between the atoms.

\section{References}

[1] Boylan, H. (1998) A Dictionary of Irish Biography. 3rd Edition, Gill and MacMillian, Dublin, 179.

[2] Crum Brown, A. (1865) On the Theory of Isomeric Compounds. Journal of the Chemical Society, 18, 230-245. http://dx.doi.org/10.1039/js8651800230

[3] Mendeleev, D. (1901) The Principles of Chemistry. Collier, New York.

[4] Abegg, R. (1904) Die Valenz und das periodische System. Zeitschrift für anorganische Chemie, 39, 330-380.

[5] Lewis, G.N. (1916) The Atom and the Molecule. Journal of the American Chemical Society, 38, 762-785. http://dx.doi.org/10.1021/ja02261a002 
[6] Langmuir, I. (1919) The Arrangement of Electrons in Atoms and Molecules. Journal of the American Chemical Society, 41, 868-934. http://dx.doi.org/10.1021/ja02227a002

[7] Gillespie, R.J. (2004) Teaching Molecular Geometry with the VSEPR Model. Journal of Chemical Education, 81, 298304. http://dx.doi.org/10.1021/ed081p298

[8] Gillespie, R.J. and Popelier, P.L.A. (2001) Chemical Bonding and Molecular Geometry. Oxford University Press, Oxford.

[9] Haynes, W.M. (1972) Structural Formula of Organic Compounds. Handbook of Chemistry and Physics, CRC Press, $53^{\mathrm{RD}}$.

[10] http://www.chemspider.com/

[11] http://www.ncbi.nlm.nih.gov/pccompound/

[12] http://en.wikipedia.org/wiki/

[13] Wurtz, A. (1859) Sur l'oxyde d'éthylène. Comptes rendus, Académie des sciences. 101-105. 